\title{
Property Improvement of Cement Emulsified Asphalt Paste Modified by Graphene Oxide
}

\author{
Yu-wei Ma, Hong-yan Zhao, Gang Li ${ }^{D}$, Zhen-jun Wang, Hua Tang, and Ai-qin Wang \\ College of Water Conservancy and Architectural Engineering, Shihezi University, Shihezi 832000, China \\ Correspondence should be addressed to Gang Li; gangli@shzu.edu.cn
}

Received 9 December 2019; Accepted 20 January 2020; Published 7 March 2020

Guest Editor: Jian Ouyang

Copyright (C) $2020 \mathrm{Yu}$-wei Ma et al. This is an open access article distributed under the Creative Commons Attribution License, which permits unrestricted use, distribution, and reproduction in any medium, provided the original work is properly cited.

\begin{abstract}
Cement emulsified asphalt paste (CEAP) is widely used as a construction and building material by combining the advantages of cement rigidity and asphalt flexibility. However, the properties of CEAP can be evidently reduced due to the addition of emulsified asphalt. In this work, graphene oxide (GO) was prepared by the Hummers method and was innovatively used to improve the workability and strength of CEAP. The viscosity of CEAP was tested by Brookfield viscometer. In addition, the effects of GO on the setting time of CEAP were studied. The adsorption between cement and asphalt with GO was tested through an ultraviolet-visible spectrophotometer and the stability of CEAP was tested by zeta potentiometer. The effects of GO on the strength of CEAP were studied. The reinforcement effects of GO on CEAP were analyzed. The results show that the viscosity of CEAP and cement hydration products can increase after adding a reasonable dosage of GO to CEAP. The setting time of CEAP first decreases and then increases with the increase of GO dosage. The adsorption and viscosity of cement and asphalt increase with the increase of GO dosage. GO can reduce CEAP stability and make the paste easier to agglomerate. The flexural strength and the compressive strength of CEAP at 28 curing days first increase and then decrease with the increase of GO dosage, but excessive GO can hinder cement hydration. The reasonable dosage of GO in CEAP can be determined as $0.06 \%$ in asphalt weight.
\end{abstract}

\section{Introduction}

Cement emulsified asphalt paste (CEAP) is widely used as a construction and building material, which has the rigidity of cement-based materials and flexibility of asphalt-based materials [1-3]. The addition of emulsified asphalt into cement can significantly improve many properties of cement material, such as rheological performance and elasticity $[4,5]$. However, the addition of emulsified asphalt can affect the cement hydration process and decrease the mechanical strength of mixtures [6-9]. At present, the main improvement technique is to add reinforcing materials, such as rebar, reinforcing fibers and inorganic binders, and slag powder.

Graphene oxide (GO) is an oxide of graphene and it is usually in the form of a solution, powder, or flake [10-12]. Its structure contains a hydroxyl group $(-\mathrm{OH})$, carboxyl group $(-\mathrm{COOH})$, epoxy group (-O-), and other hydrophilic groups [13]. GO can evenly disperse in aqueous solution due to the electrostatic repulsion between functional groups $[14,15]$.
GO has been applied in wide fields for its properties are more active than graphene. The properties of cement-based composites can be improved by the reaction between active functional groups [16]. Some researchers have found that the fluidity of cement paste was decreased and the compressive strength and flexural strength were improved with the addition of GO [17-20]. The compressive strength and flexural strength of cement-based materials are increased by $38.9 \%$ and $60.7 \%$, respectively, when the dosage of GO is $0.03 \%$ $[21,22]$. GO can influence the microscopic morphology of cement hydrates and make it regularly grow with GO as a template [23]. Go can improve the freeze-thaw cycle resistance of cement-based materials [24]. In addition, the setting time of cement can be shortened by $30 \mathrm{~min}$ when the dosage of GO is $0.05 \%$ in cement weight $[18,25]$. In addition, GO can promote the cement hydration and improve the workability of cement-based composites [26-29]. GO affects not only cement-based materials but also emulsified asphalt. GO can promote the demulsification of oil in the water 
system; the demulsification efficiency was as high as $99.94 \%$ when the dosage of GO was about $30 \mathrm{mg} / \mathrm{L}[30,31]$. GO can improve the high and low-temperature properties of asphalt and the aging resistance of asphalt $[32,33]$. In addition, GO can enhance the rutting resistance of asphalt [34].

In this work, GO was adopted to solve the problem of strength reduction of CEAP. The improvement effect of GO on CEAP was studied. The microscopic morphology of cement hydrates before and after adding GO was compared. The effect of GO on the viscosity and setting time of CEAP was tested. The influence of GO on adsorption between cement and emulsified asphalt was studied by ultravioletvisible spectrophotometer. Then, the influence of GO on the stability of CEAP was tested by the zeta potentiometer. Finally, the influence of GO on the mechanical properties of CEAP was studied. The results of this work can effectively solve the problem of low strength of CEAP and provide a theoretical basis for improving the properties of CEAP.

\section{Raw Materials and Experiments}

2.1. Raw Materials. Ordinary Portland cement was used in this work and its physical and chemical properties are shown in Table 1. Original asphalt was used to prepare emulsified asphalt and its physical and chemical properties are shown in Table 2. The properties of emulsified asphalt are shown in Table 3, which can meet with The Technical Specification for Construction of Highway Asphalt Pavements in China (JTG F40-2004).

\subsection{Experiment Methods}

2.2.1. Preparation and Characterization of GO. In this work, the Hummers method was adopted to prepare GO. The experimental process was divided into three stages by different temperatures: low-temperature stage, medium temperature stage, and high-temperature stage. The GO preparation process was as follows: (a) low-temperature stage: the flake graphene, $\mathrm{NaNO}_{3}$, and $\mathrm{KMnO}_{4}$ were added to a beaker with concentrated $\mathrm{H}_{2} \mathrm{SO}_{4}$ under stirring in a preprepared ice-water bath, and the reactive temperature was maintained at about $4^{\circ} \mathrm{C}$ for $90 \mathrm{~min}$; (b) medium temperature stage: the reaction temperature was increased to $37^{\circ} \mathrm{C}$ and stirring process was continued for $30 \mathrm{~min}$; (c) hightemperature stage: $46 \mathrm{~mL}$ water was added to the beaker and the reaction temperature was increased to $98^{\circ} \mathrm{C}$. Then, $10 \mathrm{~mL}$ $\mathrm{H}_{2} \mathrm{O}_{2}$ (30\% concentration) was slowly added in order to ensure that graphite is fully oxidized and the reaction is sustained for $20 \mathrm{~min}$. Finally, GO specimens were obtained after washing and drying. The preparation schematic diagram of GO prepared by the Hummers method is shown in Figure 1. As can be seen from Figure 2, the prepared GO samples by the Hummers method can show a lamellar structure.

2.2.2. Preparation of GO-Modified Asphalt Emulsion. The preparation chart of GO-modified asphalt emulsion is shown in Figure 3. Water $\left(60^{\circ} \mathrm{C}\right)$, emulsifier, and $\mathrm{CaCl}_{2}$ stabilizer were weighed and mixed, and then the $\mathrm{pH}$ of the mixtures was adjusted to 2-3 with hydrochloric acid. Emulsified asphalt can be obtained by pouring the prepared emulsified soap into the colloid mill and then the weighed original asphalt was slowly poured into the colloid mill. The emulsified asphalt was added to the mixer and then GO was added to obtain GO-modified asphalt emulsion. In this work, $0.02 \%, 0.04 \%, 0.06 \%, 0.08 \%$, and $0.10 \%$ GO in asphalt weight was used to prepare GO-modified asphalt emulsion, respectively. The mix proportion of GO-modified emulsified asphalt is shown in Table 4.

2.2.3. Preparation of GO-Modified CEAP. The dosage of GO-modified asphalt emulsion was $8.0 \%$ in cement weight in CEAP [28]. The water-cement ratio in CEAP was 0.38. The water consumption of emulsified asphalt was included in the total water consumption. The specimens were placed at room temperature to cure for $24 \mathrm{~h}$; then the CEAP specimens were cured for 28 days at the temperature of $20^{\circ} \mathrm{C}$ and the humidity of $90 \%$. The mix proportion of CEAP is shown in Table 5. The laboratory tests of CEAP conducted in this work are shown in Table 6.

2.2.4. ESEM Test. In this work, firstly, a small number of samples were ground into fine powders with a mortar and then pasted on the conductive adhesive. The conductivity of the samples is improved after the gold spraying treatment. Finally, the microscopy morphology of CEAP was studied with the environmental scanning electron microscope (ESEM) with observing the microscopic morphology of paste before and after adding GO with $0.06 \%$ in asphalt weight. The ESEM test was carried out at the resolution of $1 \mathrm{~nm}$ and the temperature of $20^{\circ} \mathrm{C}$.

2.2.5. Viscosity Test. According to Standard Test Methods of Bitumen and Bituminous Mixtures for Highway Engineering (JTG E20-2011), the Brookfield rotational viscometer was used to test the viscosity of CEAP with different dosages of GO at initial, $30 \mathrm{~min}$, and $60 \mathrm{~min}$ setting time. The $21 \#$ standard rotor was used in the test; the rotator speed was $50 \mathrm{r} / \mathrm{min}$ and the sample was weighed $8.5 \pm 0.5 \mathrm{~g}$ for each test. The test temperature was $20^{\circ} \mathrm{C}$ and the resolution was $0.01 \mathrm{~Pa} \cdot \mathrm{s}$. The average results of three samples from each group were adopted as the testing results.

2.2.6. Setting Time Tests. The setting process of CEAP consists of two stages, initial setting and final setting. The influences of GO on the initial and final setting time of CEAP were tested in accordance with Test Methods for Water Requirement of Normal Consistency, Setting Time and Soundness of the Portland Cement (GB/T 1346-2011).

2.2.7. Adsorption Test. Adsorption between cement and asphalt emulsion with different asphalt emulsion concentrations was measured by ultraviolet-visible spectrophotometer. The absorbance of emulsified asphalt with different 
Table 1: Properties of ordinary Portland cement.

\begin{tabular}{|c|c|c|c|c|c|c|c|}
\hline \multicolumn{2}{|c|}{ Setting time (min) } & \multicolumn{2}{|c|}{ Flexural strength $(\mathrm{MPa})$} & \multicolumn{2}{|c|}{$\begin{array}{c}\text { Compressive strength } \\
(\mathrm{MPa})\end{array}$} & \multicolumn{2}{|c|}{$\begin{array}{l}\text { Main chemical } \\
\text { composition (\%) }\end{array}$} \\
\hline Initial & Final & 3 days & 28 days & 3 days & 28 days & $\mathrm{CaO}$ & $\mathrm{SiO}_{2}$ \\
\hline 140 & 271 & 3.8 & 6.9 & 18.1 & 45.2 & 60.1 & 20.8 \\
\hline
\end{tabular}

TABle 2: Properties of original asphalt.

\begin{tabular}{lcccccc}
\hline $\begin{array}{l}\text { Penetration } \\
\left(25^{\circ} \mathrm{C}, 0.1 \mathrm{~mm}\right)\end{array}$ & Ductility $\left(15^{\circ} \mathrm{C}, \mathrm{cm}\right)$ & Softening point $\left({ }^{\circ} \mathrm{C}\right)$ & Solubility $(\%)$ & Flash Point $\left({ }^{\circ} \mathrm{C}\right)$ & Density $\left(\mathrm{g} / \mathrm{cm}^{3}\right)$ & Wax content $(\%)$ \\
\hline 65.0 & 15.0 & 45.2 & 99.6 & 265.0 & 1.03 \\
\hline
\end{tabular}

TAble 3: Properties of asphalt emulsion.

\begin{tabular}{|c|c|c|c|c|c|c|}
\hline \multirow[t]{2}{*}{$\begin{array}{l}\text { Evaporation } \\
\text { residue content (\%) }\end{array}$} & \multirow[t]{2}{*}{$\begin{array}{c}\text { Residue on } 1.18 \mathrm{~mm} \\
\text { sieve }(\%)\end{array}$} & \multirow[t]{2}{*}{$\begin{array}{l}\text { Penetration of residue } \\
\left(25^{\circ} \mathrm{C}, 0.1 \mathrm{~mm}\right)\end{array}$} & \multirow[t]{2}{*}{$\begin{array}{l}\text { Softening point of } \\
\text { residue }\left({ }^{\circ} \mathrm{C}\right)\end{array}$} & \multirow[t]{2}{*}{$\begin{array}{c}\text { Ductility of } \\
\text { residue }\left(\mathrm{cm}, 15^{\circ} \mathrm{C}\right)\end{array}$} & \multicolumn{2}{|c|}{$\begin{array}{c}\text { Storage } \\
\text { stability } \\
\left(\%, 25^{\circ} \mathrm{C}\right)\end{array}$} \\
\hline & & & & & $1 \mathrm{~d}$ & $5 \mathrm{~d}$ \\
\hline 60.9 & 0.04 & 77.1 & 45.7 & 49.8 & 0.4 & 2.2 \\
\hline
\end{tabular}

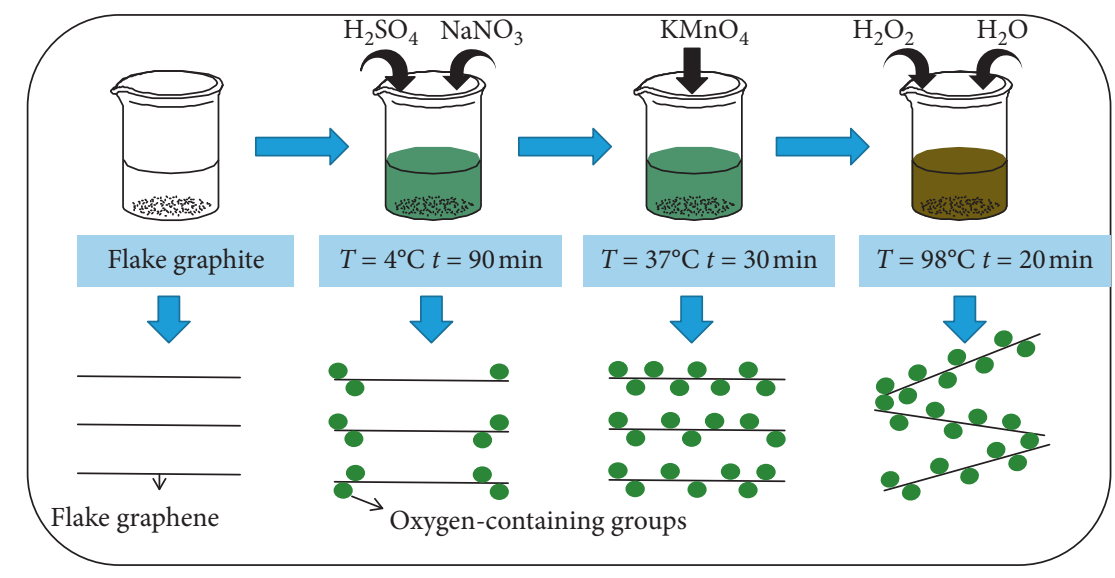

FIGURE 1: Schematic diagram of GO preparation by Hummers method.

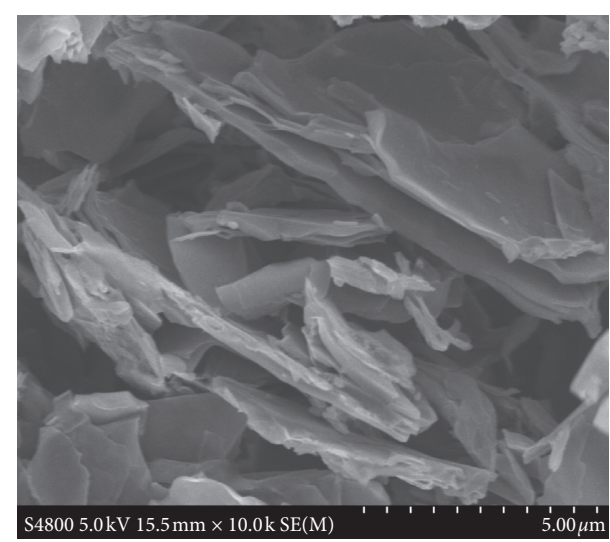

FIGURE 2: ESEM picture of graphene oxide.

concentrations was measured at $750 \mathrm{~nm}$ wavelength [35]. When the function had fitted, the emulsified asphalt was firstly prepared and the absorbance was measured to obtain its concentration. Then, cement was added to the emulsion of emulsified asphalt. The supernatant fluid concentration was tested after the paste was stirred and allowed to stand for 30 minutes. Finally, the adsorption rate between cement and emulsified asphalt can be calculated by

$$
D=\frac{10 m_{1}}{m_{2}} \times\left(c_{1}-c_{2}\right),
$$

where $D$ is the adsorption rate between cement and asphalt emulsion $(\mathrm{mg} / \mathrm{g}), m_{1}$ is emulsified asphalt weight $(\mathrm{g}), m_{2}$ is cement weight $(\mathrm{g}), c_{1}$ is the initial concentration of emulsified asphalt (\%), and $c_{2}$ is the concentration of emulsified asphalt at one moment (30 min, in this work) (\%). The adsorption test process is shown in Figure 4.

2.2.8. Zeta Potential Test. The stability of CEAP suspension was evaluated by measuring the surface charge of particles. CEAP with different GO dosages was used to obtain zeta potential by zeta potential test. The effect of different GO dosages on the stability of CEAP was also analyzed. The potential test was carried out at the temperature of $20^{\circ} \mathrm{C}$. 


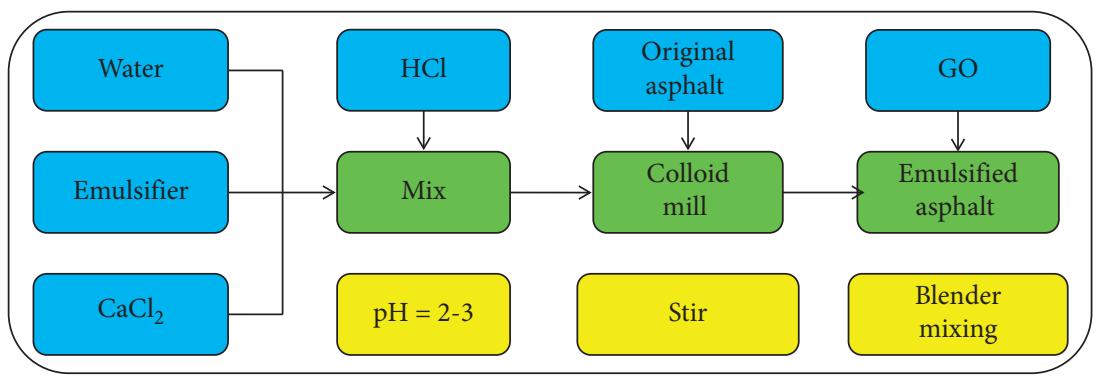

Figure 3: Preparation chart of GO-modified asphalt emulsion.

TABle 4: Mix proportion of GO-modified emulsified asphalt.

\begin{tabular}{lccc}
\hline GO dosage $(\%)$ & Original asphalt $(\mathrm{g})$ & Water $(\mathrm{g})$ & GO dosage $(\mathrm{g})$ \\
\hline 0.02 & 600 & 400 & 0.12 \\
0.04 & 600 & 400 & 0.24 \\
0.06 & 600 & 400 & 0.36 \\
0.08 & 600 & 400 & 0.48 \\
0.10 & 600 & 400 & 0.60 \\
\hline
\end{tabular}

TABle 5: The mix proportion of CEAP.

\begin{tabular}{|c|c|c|c|c|c|}
\hline \multirow{2}{*}{ GO (\%) } & \multirow{2}{*}{ Cement (g) } & \multirow{2}{*}{ Water $(\mathrm{g})$} & \multicolumn{3}{|c|}{ GO-modified emulsified asphalt } \\
\hline & & & Asphalt (g) & Water $(\mathrm{g})$ & $\mathrm{GO}(\mathrm{g})$ \\
\hline 0.02 & 1350 & 469.8 & 64.8 & 43.2 & 0.013 \\
\hline 0.04 & 1350 & 469.8 & 64.8 & 43.2 & 0.026 \\
\hline 0.06 & 1350 & 469.8 & 64.8 & 43.2 & 0.039 \\
\hline 0.08 & 1350 & 469.8 & 64.8 & 43.2 & 0.052 \\
\hline 0.10 & 1350 & 469.8 & 64.8 & 43.2 & 0.065 \\
\hline
\end{tabular}

TABLE 6: The laboratory tests of CEAP.

\begin{tabular}{lcc}
\hline Laboratory tests & Properties & Objectives \\
\hline ESEM test & Micromorphology & Effects of GO on the morphology of the paste \\
Viscosity test & Viscosity & Setting time \\
Setting time test & Adsorption & Effects of GO on the adsorption between cement and asphalt \\
Adsorption test & Stability & Effects of GO on cement hydration and asphalt demulsification \\
Zeta potential test & Effects of GO on adsorption between cement and asphalt \\
Mechanical property tests & Mechanical properties & Effects of GO on the stability of the paste
\end{tabular}

2.2.9. Mechanical Property Tests. The specimens with sizes of $40 \mathrm{~mm} \times 40 \mathrm{~mm} \times 160 \mathrm{~mm}$ were prepared for strength tests, such as flexural strength and compressive strength. The flexural strength and compressive strength of CEAP were tested according to Method of Testing Cements-Determination of Strength (GB/T17671-1999). The flexural strength and the compressive strength of CEAP were tested three times and six times, respectively, and the average value was adopted as the test result.

\section{Results and Discussion}

3.1. Viscosity Test Results. The adsorption between cement and asphalt is closely related to the stability of CEAP. The stronger the adsorption between cement and asphalt, the faster hydration of cement and the demulsification of emulsified asphalt, and the greater the viscosity of the paste. Therefore, the adsorption between cement and asphalt can be characterized by testing the viscosity of the paste. The adsorptions between cement and asphalt with different GO dosages can be reflected by the viscosity of CEAP.

Figure 5 shows the viscosity of paste increases with the increase of GO dosage at initial, $30 \mathrm{~min}$, and $60 \mathrm{~min}$ setting time. The phenomenon can be explained by the following reasons. GO can be evenly dispersed in the paste. GO can result in the aggregation of free water and promote cement hydration. The addition of GO can also absorb the free water, which can result in the difference of the free water concentration in the paste and promote the demulsification of emulsified asphalt. In addition, the asphalt can wrap the 


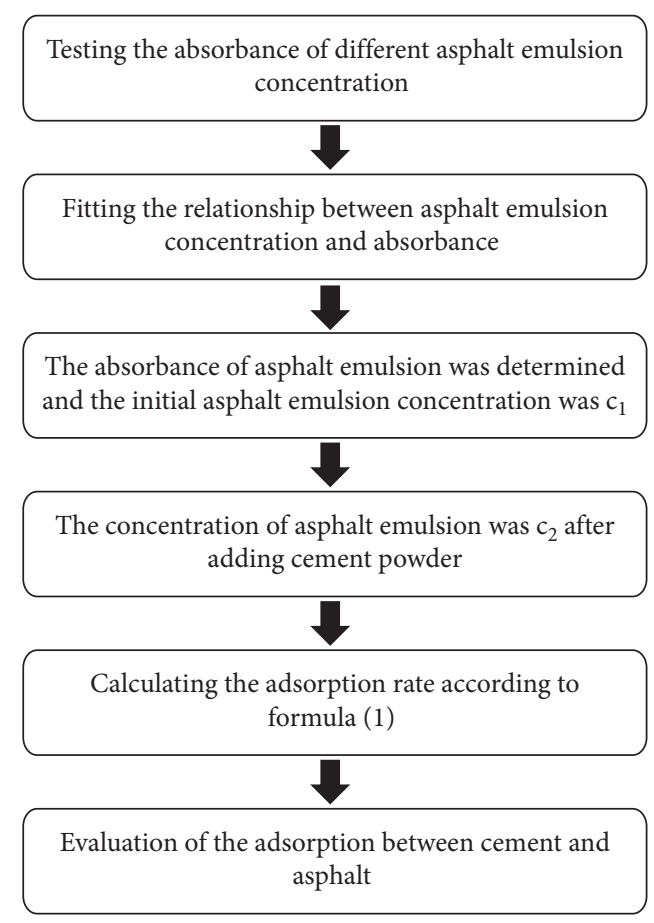

FIGURE 4: The process for the adsorption test.

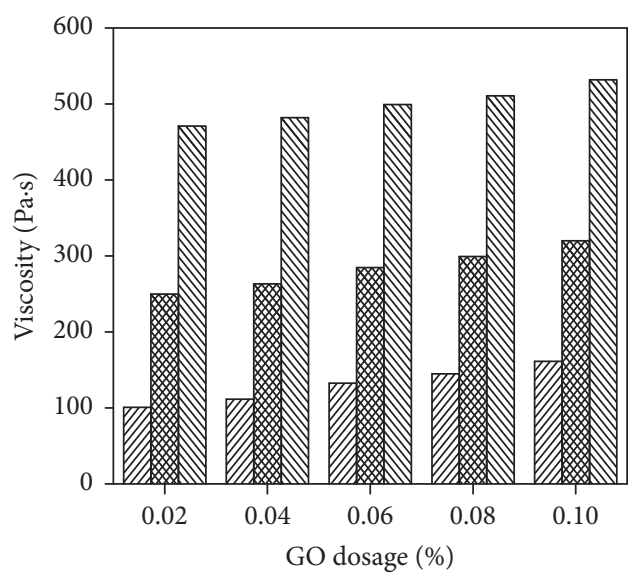

$$
\begin{aligned}
& \text { IIItA Initial } \\
& 30 \mathrm{~min} \\
& \text { MIV } 60 \mathrm{~min}
\end{aligned}
$$

Figure 5: Viscosity of CEAP with different GO dosage.

cement hydration products, which can form a complex spatial network structure. Therefore, the viscosity of CEAP is increased.

3.2. Setting Time Test Results. The setting time has an important influence on the strength development of CEAP. When the flexible emulsified asphalt is added to the rigid cement paste, the setting time of cement paste can be increased. However, the addition of GO can shorten the setting time of cement-based materials. Therefore, the effect of GO on the setting time of CEAP was studied.

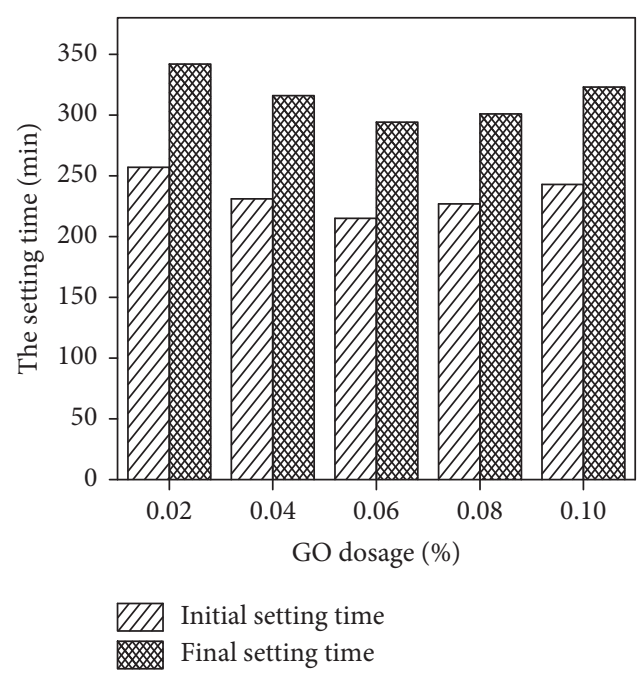

FIGURE 6: Setting time of CEAP with different GO dosages.

The setting time test results of CEAP with different dosages of GO are shown in Figure 6. It shows that the initial and final setting time of CEAP first decreases and then increases with the increase of GO dosage. Some reasons can be used to explain this phenomenon. The effect of GO on cement is higher than that of GO on emulsified asphalt when the dosage of GO is little. The free water in the paste is agglomerated and the cement hydration is promoted due to lots of hydrophilic groups on the surface of GO. Consequently, the setting time of CEAP is decreased. However, the effect of GO on emulsified asphalt is higher than that of GO on cement when the dosage of GO increases (more than $0.06 \%$ in asphalt weight). The difference in free water concentration caused by GO can promote emulsified asphalt demulsification, hinder cement hydration, and increase the setting time of CEAP.

3.3. Absorptions in GO-Modified CEAP. Figure 7 shows the absorbance results of CEAP at different asphalt emulsion concentrations. The concentration of asphalt emulsion can be obtained from Figure 7 through testing the absorbance of the solution. The absorbance increases gradually with the increase of asphalt emulsion concentration. It is mainly due to the increase of asphalt emulsion concentration, the increase of asphalt concentration, and the thickness of asphalt film in the solution. At the same time, the absorbance increased with the increase of GO dosage. This phenomenon can be explained by these reasons. On the one hand, the increase of GO dosage can result in the increase of solution concentration; on the other hand, GO can promote the demulsification of emulsified asphalt and reduce the intensity of refraction light. As a result, the absorbance between cement and emulsified asphalt increases.

The fitting curve expression of emulsified asphalt with different GO dosages and the corresponding absorbance values are shown in Table 7 . It shows that the $\left(c_{1}-c_{2}\right)$ value of CEAP increases with the increase of GO dosage. In other words, the adsorption of cement and asphalt increases with the increase of GO dosage. Therefore, the addition of GO is 


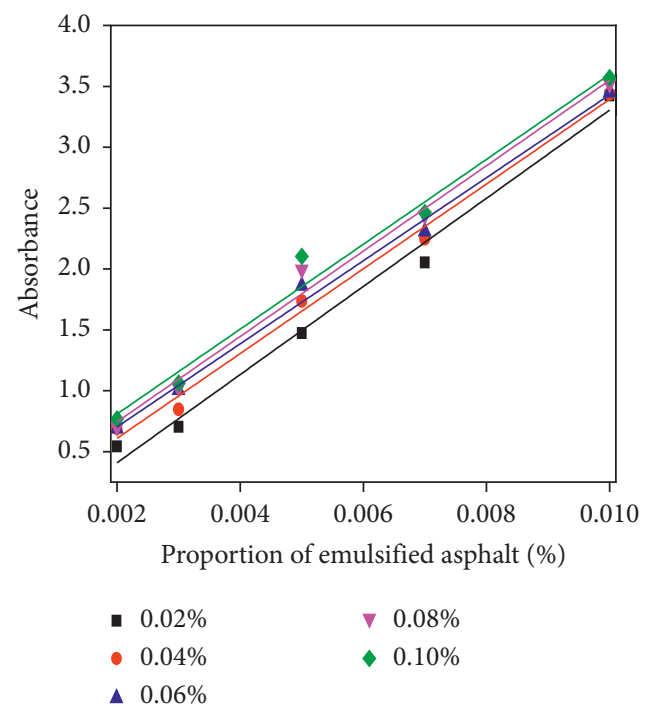

FIGURe 7: Absorbance results of CEAP with different emulsified asphalt concentrations.

TABLE 7: Effects of GO on absorption between cement and emulsified asphalt.

\begin{tabular}{|c|c|c|c|c|c|c|}
\hline \multirow{2}{*}{ GO dosage $(\%)$} & \multirow{2}{*}{ Regression curve } & \multicolumn{2}{|c|}{ Absorbance } & \multicolumn{2}{|c|}{ Proportion of emulsified asphalt (\%) } & \multirow{2}{*}{$\left(c_{1}-c_{2}\right)(\%$} \\
\hline & & Mixed cement & Unmixed cement & Mixed cement & Unmixed cement & \\
\hline 0.02 & $y=362.08 x-0.32$ & 0.762762 & 0.735821 & 0.00299 & 0.00292 & 0.00008 \\
\hline 0.04 & $y=348.13 x-0.09$ & 0.859808 & 0.811187 & 0.00273 & 0.00259 & 0.00014 \\
\hline 0.06 & $y=341.08 x+0.02$ & 0.997323 & 0.875902 & 0.00287 & 0.00251 & 0.00036 \\
\hline 0.08 & $y=350.74 x+0.04$ & 1.103588 & 0.902976 & 0.00303 & 0.00246 & 0.00057 \\
\hline 0.10 & $y=348.66 x+0.11$ & 1.342773 & 1.130586 & 0.00354 & 0.00293 & 0.00062 \\
\hline
\end{tabular}

helpful to the combination of asphalt and cement. The interaction among GO, cement, and emulsified asphalt is shown in Figure 8. GO has numbers of active functional groups on its surface, which can generate physical and chemical reactions between $\mathrm{GO}$ and asphalt molecules and increase the combination between GO and asphalt. In addition, cement hydrates can grow around with GO as the template and the combination between GO and cement hydrates can increase [36]. Therefore, GO is equivalent to a bridge connecting cement and emulsified asphalt to strengthen the combination between cement and asphalt. Consequently, GO can increase the adsorption between cement and asphalt.

3.4. Stability of CEAP. Workability stability is an important parameter to evaluate the performance of CEAP. The potential of CEAP with different GO dosages was tested by zeta potentiometer and the results are shown in Figure 9. It shows that the zeta potential value decreases with the increase of GO dosage. It indicates that the stability of CEAP is decreased with the increase of GO dosage and the paste is more condensable. Some reasons can explain this phenomenon. Firstly, for the presence of oxygen-containing functional groups on the surface of GO, it is easy to absorb ions or cement particles with opposite charges in CEAP to generate agglomeration and flocculation, which can reduce the stability of the paste. Secondly, GO can adsorb water molecules and promote the cement hydration process in cement materials.

At the same time, GO also can adsorb ions with opposite charges in cement to cause agglomeration and flocculation of cement particles. When GO adsorbs water molecules, hydration products preferentially form polyhedral structure. Then, the cement hydration products grow around with the polyhedral structure as the template. The grown cement hydration products can shield the attraction of GO to opposite charges and can reduce the agglomeration and flocculation of the paste. Finally, GO can cause agglomeration and flocculation of cement particles and can affect the cement hydration, which can decrease the workability stability of CEAP. In other words, CEAP is easier to agglomerate.

3.5. Mechanical Properties of CEAP. Flexural strength and compressive strength test results of CEAP cured at 28 days are shown in Figure 10. It shows that the flexural and compressive strength of CEAP first increases and then decreases with the increase of GO dosage. The addition of GO can improve the flexural and compressive strength of CEAP. The flexural and compressive strength of CEAP can reach the maximum value when the dosage of GO is $0.06 \%$ in asphalt weight.

The microstructures of CEAP before and after adding $0.06 \%$ GO were observed by ESEM and the results are shown 


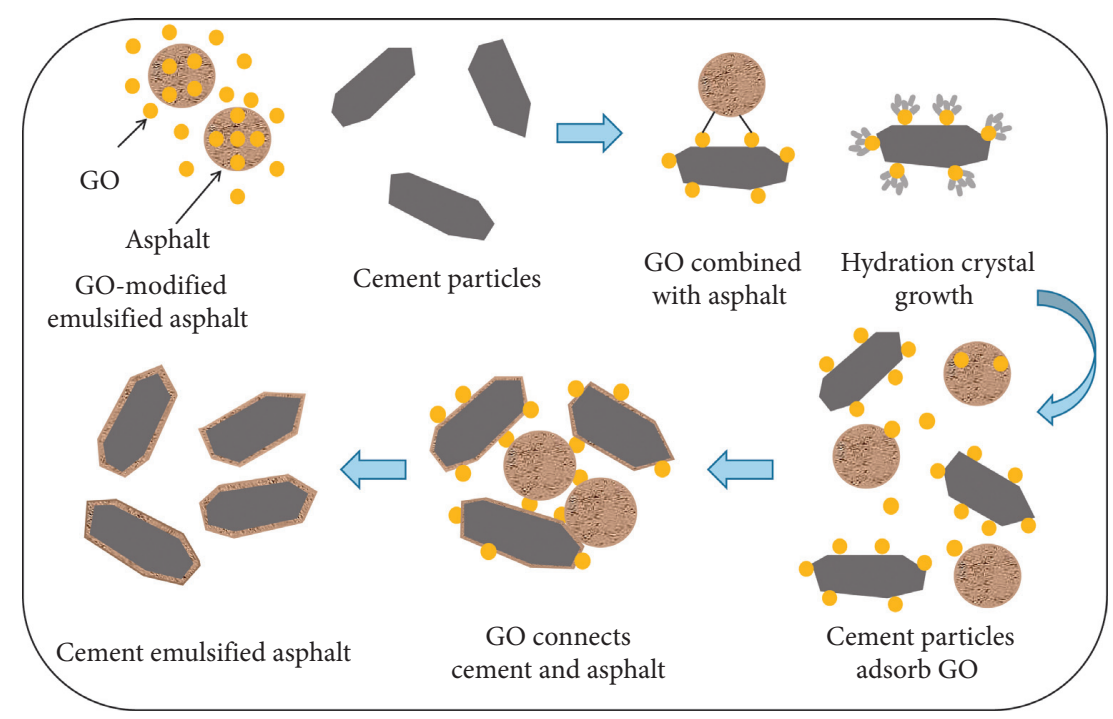

FIgURE 8: Diagrammatic sketch of absorption in CEAP.

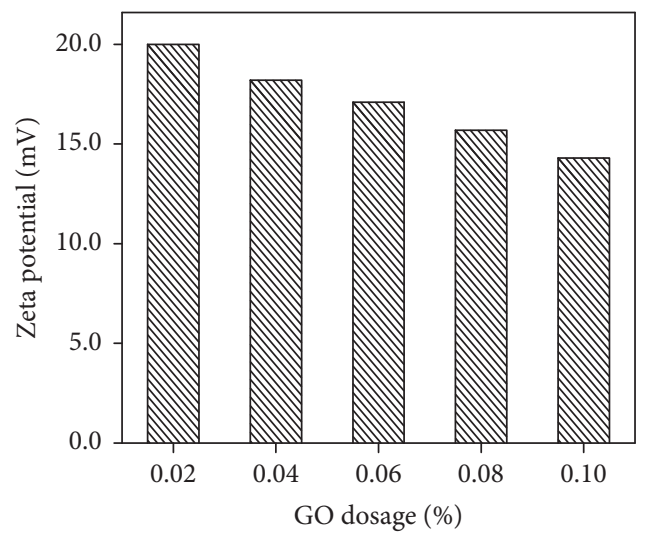

FIgURE 9: Zeta potential of CEAP with different GO dosages.

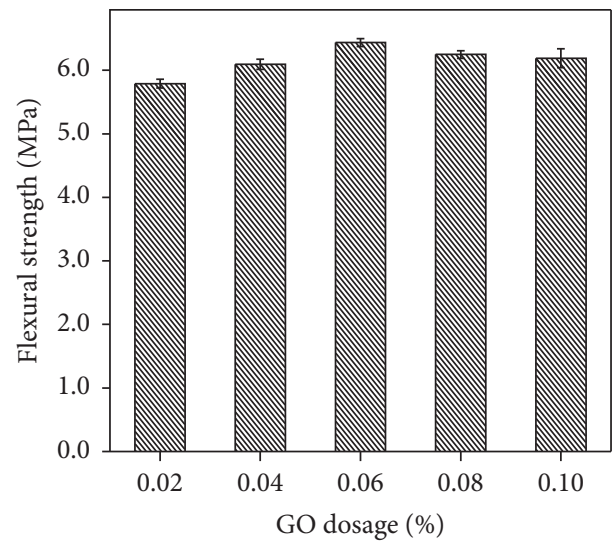

(a)

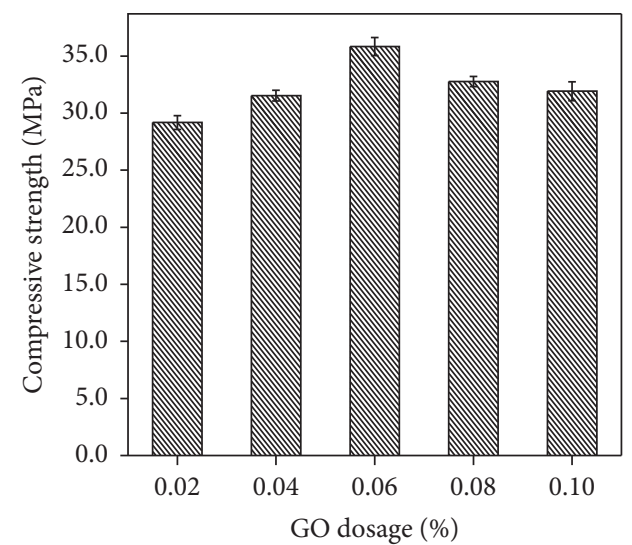

(b)

Figure 10: Effects of GO on the strength of CEAP. (a) Flexural strength. (b) Compressive strength.

in Figure 11. In contrast to the microscopic morphology of CEAP before and after adding GO, it is found that there are some cracks and fewer hydration products. A part of cement particles is wrapped with asphalt and there exists loose structure in CEAP without GO. However, the number of cracks decreases, the hydration products increases, and the 


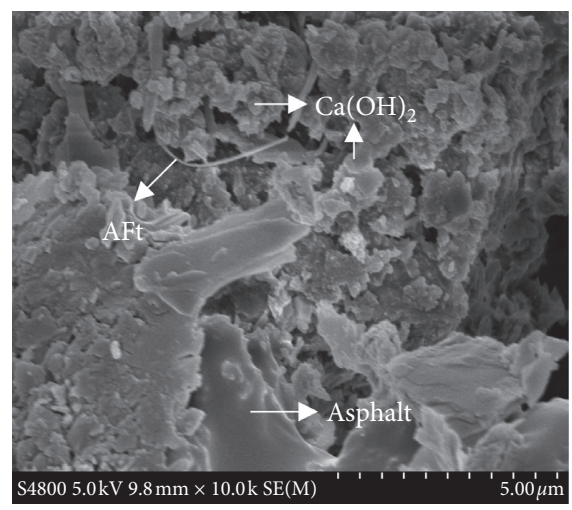

(a)

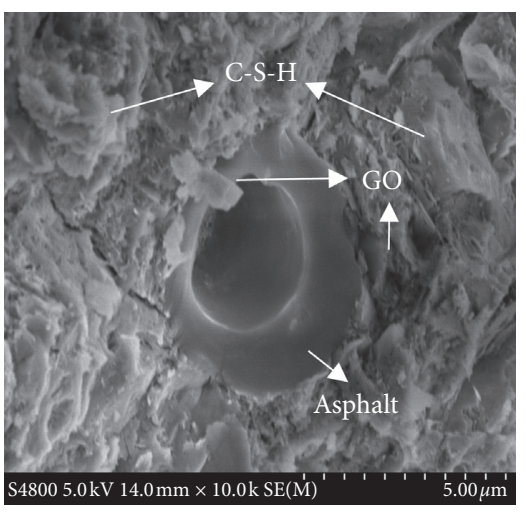

(b)

Figure 11: ESEM pictures of CEAP: (a) without GO and (b) with GO.

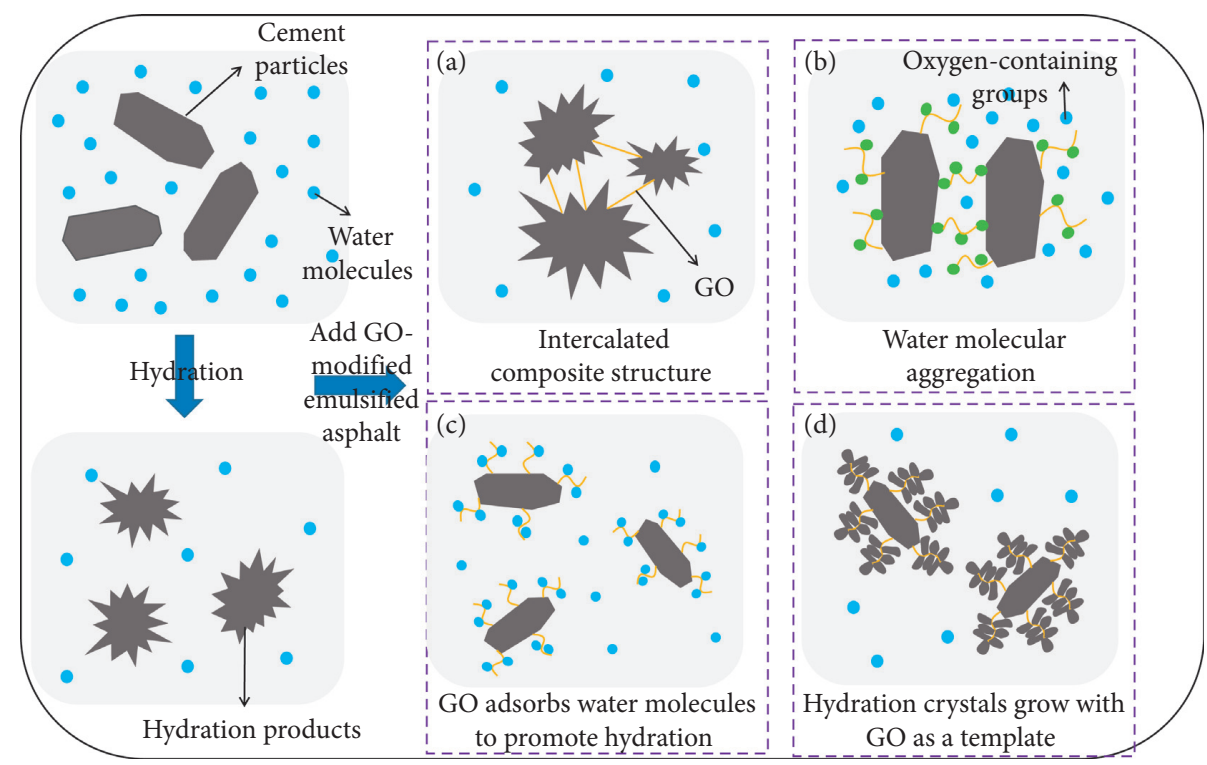

Figure 12: Diagrammatic sketch of interaction between GO and cement emulsified asphalt.

structure becomes denser when GO is added in CEAP. The reason is that GO can promote cement hydration and increase the cement hydration products in CEAP. Therefore, GO can improve the flexural and compressive strength of CAEP.

3.6. Mechanism of GO-Modified CEAP. GO is easy to form intercalation structure with cement hydration products, which can increase the properties of CEAP. The reasons are that the strong oxidant gradually adsorbs on the edge of graphite, oxidizes, and intercalates the edge part in the lowtemperature reaction. A part of intermolecular forces is gradually destroyed and the oxygen-containing functional groups are generated. The reaction temperature is increased after the low-temperature oxidation. The oxidation and intercalation ability of strong oxidant is gradually enhanced with the increase of reaction temperature. More oxygencontaining functional groups are generated between graphite sheets, which put forward the foundation for the stripping of graphite sheets. The residual intermolecular forces are destroyed after the addition of deionized water and hydrogen peroxide in the high-temperature stage, which can make the graphite sheets strip " to " which will peel off the graphite sheets. The increase of the active sites of GO will increase the reaction with cement paste.

In addition, it is easy to form an intercalated composite structure with hydration products to increase the properties of CEAP, due to the high strength and flexibility of GO. The electrostatic interaction between oxygen-containing groups on the GO surface and cement particles results in agglomeration and flocculation. The free water of the system is lost because this function requires a large amount of free water. Therefore, the friction between the cement particles is increased, the fluidity of CEAP is decreased, the setting time is shortened, and the strength of CEAP is increased. GO adsorbs water molecules in cement paste after adding GO. It leads to aggregation of free water and promotes cement hydration reaction [16]. In addition, the oxygen-containing functional groups of GO can react with the reactive groups 


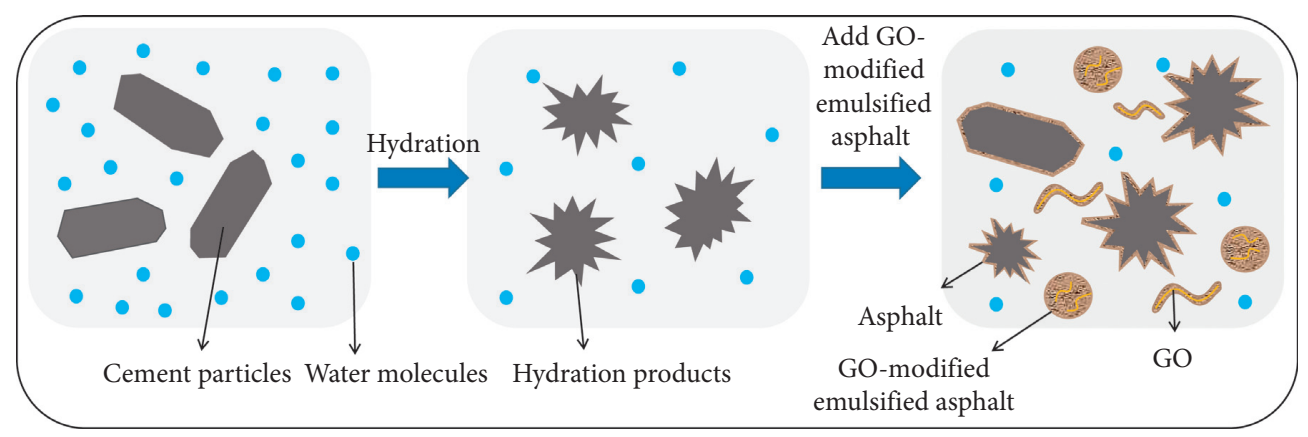

FIGURE 13: Diagrammatic sketch of GO influence on CEAP.

$\mathrm{C}_{3} \mathrm{~S}, \mathrm{C}_{3} \mathrm{~A}, \mathrm{C}_{2} \mathrm{~S}$, and $\mathrm{C}_{4} \mathrm{AF}$ in the cement to form hydrated crystals in the process of the hydration process. The hydration products are preferentially fixed on the GO sheet and the cement hydration products grow continuously around GO template. Interwoven, cross-linked, dense, and uniform network structure is formed, which can increase the properties of CEAP [21].

The interaction between GO and cement emulsified asphalt binders in CEAP is shown in Figure 12. Cement hydration can produce amounts of calcium hydroxide, which causes the cement paste to be alkaline. GO is agglomerated due to the difference of $\mathrm{pH}$ value in the alkaline paste, which can result in the uneven dispersion of GO in CEAP and can affect the mechanical properties of the paste. However, GO can be evenly dispersed in emulsified asphalt due to the existence of hydrophilic groups on the surface of GO.

However, the properties of CEAP decrease with the increase of GO dosage again. As can be seen from Figure 13, the nonhydrated cement particles and hydration products are covered by GO, which can hinder the cement hydration and reduce the properties of CEAP. In addition, redundant GO is encapsulated in asphalt particles, which cannot make contact with cement and reduce the strengthening effect of GO on CEAP. Therefore, the mechanical properties of CEAP are reduced.

\section{Conclusions and Recommendations}

In this work, GO was prepared by the Hummers method and it was adopted in emulsified asphalt to prepare GO-modified emulsified asphalt. Then, the GO-modified asphalt emulsion was used to prepare CEAP. The effects of GO on the viscosity and setting time of CEAP were tested and analyzed. The influences of GO on properties of CEAP were analyzed with microcharacterizations, such as an ultraviolet-visible spectrophotometer, zeta potentiometer, and ESEM. In addition, the strengthening effect of GO on CEAP was tested. Finally, the mechanism of GO-modified CEAP was analyzed. The following conclusions are drawn:

(1) The viscosity and setting time of CEAP with different dosage GO was tested. The results show that the viscosity of CEAP increases with the increase of the GO dosage. The setting time of CEAP first decreases and then increases with the increase of GO dosage. Therefore, a small amount of GO can promote cement hydration. However, it can also promote the emulsified asphalt demulsification and hinder the cement hydration with the increase of GO dosage.

(2) The adsorption between cement and emulsified asphalt with GO was tested by an ultraviolet-visible spectrophotometer. The results show that the adsorption between cement and emulsified asphalt increases with the increase of the GO dosage. Therefore, the addition of GO is beneficial to form reinforcing binders by promoting the combination of cement and asphalt.

(3) The zeta potential decreases with the increase of the GO dosage, which indicates that the stability of CEAP decreases. GO can adsorb the oppositely charged particles in the paste, which can result in agglomeration and flocculation of cement particles. Therefore, GO can reduce the stability of the CEAP and make the paste easier to agglomerate.

(4) The flexural strength and compressive strength of CEAP first increase and then decrease with the increase of the GO dosage. GO can improve the strength of CEAP through promoting the growth of cement hydration products. However, the flexural strength and compressive strength decrease because excessive GO can result in the emulsified asphalt demulsification and then can hinder the cement hydration.

(5) GO with suitable dosage can improve the viscosity, setting time, and stability of CEAP and increase the strength of harden CEAP. According to the test results of the workability and mechanical properties of CEAP, the reasonable dosage of GO in CEAP is determined and recommended as $0.06 \%$ in asphalt weight.

\section{Data Availability}

All data generated or analyzed during this study are included in this article.

\section{Conflicts of Interest}

There are no conflicts of interest regarding the publication of this paper. 


\section{Acknowledgments}

This work was supported by the Science and Technology Development Project of the Xinjiang Production and Construction Corps (no. 2019AB013).

\section{References}

[1] X.-t. Qin, S.-y. Zhu, S.-f. Chen, X. Li, and H.-b. Dou, "Comparative study on the deformation behaviors of cement emulsified asphalt mortars," Materials and Structures, vol. 48, no. 10, pp. 3241-3247, 2015.

[2] T. Rutherford, Z. Wang, X. Shu, B. Huang, and D. Clarke, "Laboratory investigation into mechanical properties of cement emulsified asphalt mortar," Construction and Building Materials, vol. 65, pp. 76-83, 2014.

[3] Z. Wang, N. Dai, X. Wang, J. Zhang, and H. Guo, "Laboratory investigation on effects of microwave heating on early strength of cement bitumen emulsion mixture," Construction and Building Materials, vol. 236, Article ID 117439, 2020.

[4] Y. Du, L. Kong, and T. Wei, "Laboratory investigation into early-age strength improvement of cold recycled asphalt mixture containing asphalt emulsion and cement," Advances in Civil Engineering, vol. 2019, pp. 1-10, 2019.

[5] S. Al-Merzah, S. Al-Busaltan, and A. N. Hassan, "Characterizing cold bituminous emulsion mixtures comprised of palm leaf ash," Journal of Materials in Civil Engineering, vol. 31, no. 6, Article ID 04019069, 2019.

[6] X. Yan, Z. Wang, M. Rao, and M. Li, "Investigation of cementemulsified asphalt in plastic concrete," Advances in Materials Science and Engineering, vol. 2018, Article ID 3929682, 13 pages, 2018.

[7] T. Ma, H. Wang, Y. Zhao, X. Huang, and Y. Pi, "Strength mechanism and influence factors for cold recycled asphalt mixture," Advances in Materials Science and Engineering, vol. 2015, Article ID 181853, 10 pages, 2015.

[8] J. Ouyang, W. Yang, J. Chen, B. Han, Y. Meng, and T. Tang, "Effect of superplasticizer and wetting agent on the pavement properties of cold recycled mixture with bitumen emulsion and cement," Advances in Materials in Civil Engineering, vol. 2020, Article ID 6251653, 11 pages, 2020.

[9] J. Ouyang, J. Zhao, and Y. Tan, "Modeling mechanical properties of cement asphalt emulsion mortar with different asphalt to cement ratios and temperatures," Journal of $M a$ terials in Civil Engineering, vol. 30, no. 10, Article ID 04018263, 2018.

[10] L. Chen, Y. Li, Q. Du et al., "High performance agar/graphene oxide composite aerogel for methylene blue removal," Carbohydrate Polymers, vol. 155, pp. 345-353, 2017.

[11] M. S. Usman, M. Z. Hussein, S. Fakurazi, and F. F. A. Saad, "Gadolinium-based layered double hydroxide and graphene oxide nano-carriers for magnetic resonance imaging and drug delivery," Chemistry Central Journal, vol. 11, no. 1, pp. 275-278, 2017.

[12] R. Wang, J. Yue, R. Li, and Y. Sun, "Evaluation of aging resistance of asphalt binder modified with graphene oxide and carbon nanotubes," Journal of Materials in Civil Engineering, vol. 31, no. 11, Article ID 04019274, 2019.

[13] V. Kumar Gupta, S. Agarwal, M. Asif, A. Fakhri, and N. Sadeghi, "Application of response surface methodology to optimize the adsorption performance of a magnetic graphene oxide nanocomposite adsorbent for removal of methadone from the environment," Journal of Colloid and Interface Science, vol. 497, pp. 193-200, 2017.
[14] V. Loryuenyong, K. Totepvimarn, P. Eimburanapravat, W. Boonchompoo, and A. Buasri, "Preparation and characterization of reduced graphene oxide sheets via water-based exfoliation and reduction methods," Advances in Materials Science and Engineering, vol. 2013, Article ID 923403, 5 pages, 2013.

[15] L. Liu, L. Wang, J. Gao, J. Zhao, X. Gao, and Z. Chen, "Amorphous structural models for graphene oxides," Carbon, vol. 50, no. 4, pp. 1690-1698, 2012.

[16] A. Mohammed, J. G. Sanjayan, W. H. Duan, and A. Nazari, "Incorporating graphene oxide in cement composites: a study of transport properties," Construction and Building Materials, vol. 84, pp. 341-347, 2015.

[17] F. Babak, H. Abolfazl, R. Alimorad, and G. Parviz, "Preparation and mechanical properties of graphene oxide: cement nanocomposites," The Scientific World Journal, vol. 2014, Article ID 276323, 10 pages, 2014.

[18] Q. Wang, J. Wang, C.-X. Lu, B.-w. Liu, K. Zhang, and C.-z. Li, "Influence of graphene oxide additions on the microstructure and mechanical strength of cement," New Carbon Materials, vol. 30, no. 4, pp. 349-356, 2015.

[19] Z. Lu, D. Hou, L. Meng, G. Sun, L. Cong, and Z. Li, "Mechanism of cement paste reinforced by graphene oxide/ carbon nanotubes composites with enhanced mechanical properties," RSC Advances, vol. 5, no. 5, pp. 100598-100605, 2015.

[20] W. Li, X. Li, S. J. Chen et al., "Effects of graphene oxide on early-age hydration and electrical resistivity of Portland cement paste," Construction and Building Materials, vol. 136, pp. 506-514, 2017.

[21] S. Lv, Y. Ma, C. Qiu, T. Sun, J. Liu, and Q. Zhou, "Effect of graphene oxide nanosheets of microstructure and mechanical properties of cement composites," Construction and Building Materials, vol. 49, pp. 121-127, 2013.

[22] K. Gong, Z. Pan, A. H. Korayem et al., "Reinforcing effects of graphene oxide on portland cement paste," Journal of Materials in Civil Engineering, vol. 27, no. 2, Article ID A4014010, 2014.

[23] L. Lu and D. Ouyang, "Properties of cement mortar and ultrahigh strength concrete incorporating graphene oxide nanosheets," Nanomaterials, vol. 7, no. 7, p. 187, 2017.

[24] A. Mohammed, J. G. Sanjayan, W. H. Duan, and A. Nazari, "Graphene oxide impact on hardened cement expressed in enhanced freeze-thaw resistance," Journal of Materials in Civil Engineering, vol. 28, no. 9, Article ID 04016072, 2016.

[25] Z. Lu, D. Hou, H. Ma, T. Fan, and Z. Li, "Effects of graphene oxide on the properties and microstructures of the magnesium potassium phosphate cement paste," Construction and Building Materials, vol. 119, pp. 107-112, 2016.

[26] W.-J. Long, J.-J. Wei, H. Ma, and F. Xing, "Dynamic mechanical properties and microstructure of graphene oxide nanosheets reinforced cement composites," Nanomaterials, vol. 7, no. 12, p. 407, 2017.

[27] X. Li, C. Li, Y. Liu et al., "Improvement of mechanical properties by incorporating graphene oxide into cement mortar," Mechanics of Advanced Materials and Structures, vol. 25, no. 15-16, pp. 1313-1322, 2016.

[28] O. Xu, Z. Wang, and R. Wang, "Effects of aggregate gradations and binder contents on engineering properties of cement emulsified asphalt mixtures," Construction and Building Materials, vol. 135, pp. 632-640, 2017.

[29] W. Li, X. Li, S. J. Chen, G. Long, Y. M. Liu, and H. Wen, "Effects of nanoalumina and graphene oxide on early-age hydration and mechanical properties of cement paste," 
Journal of Materials in Civil Engineering, vol. 29, no. 9, Article ID 04017087, 2017.

[30] J. Liu, X. Li, W. Jia, Z. Li, Y. Zhao, and S. Ren, "Demulsification of crude oil-in-water emulsions driven by graphene oxide nanosheets," Energy \& Fuels, vol. 29, no. 7, pp. 4644-4653, 2015.

[31] J. Liu, H. Wang, X. Li, W. Jia, Y. Zhao, and S. Ren, "Recyclable magnetic graphene oxide for rapid and efficient demulsification of crude oil-in-water emulsion," Fuel, vol. 189, pp. 79-87, 2017.

[32] W. Zeng, S. Wu, L. Pang, Y. Sun, and Z. Chen, "The utilization of graphene oxide in traditional construction materials: Asphalt," Materials, vol. 10, no. 1, p. 48, 2017.

[33] S. Wu, Z. Zhao, Y. Li, L. Pang, S. Amirkhanian, and M. Riara, "Evaluation of aging resistance of graphene oxide modified asphalt," Applied Sciences, vol. 7, no. 7, p. 702, 2017.

[34] K. Liu, K. Zhang, and X. Shi, "Performance evaluation and modification mechanism analysis of asphalt binders modified by graphene oxide," Construction and Building Materials, vol. 163 , pp. $880-889,2018$.

[35] Z. Wang, H. Wang, T. Zhang, and C. Xu, "Investigation on absorption performance between cement and emulsified asphalt with UV-Vis spectrophotometer," Construction and Building Materials, vol. 136, pp. 256-264, 2017.

[36] M. Wang, R. Wang, H. Yao, Z. Wang, and S. Zheng, “Adsorption characteristics of graphene oxide nanosheets on cement," RSC Advances, vol. 6, no. 68, pp. 63365-63372, 2016. 